\title{
Shear wave elastography (SWE) may be more sensitive and more precise than transient elastography (TE) in predicting significant fibrosis in CHB patients $₫ A$ prospective comparative study
}

\section{Tiantian Yao}

Peking University First Hospital

Jing Pan

Peking University First Hospital

Jiandan Qian

Peking University First Hospital

Hao Cheng

Peking University First Hospital

Yan Wang ( $\nabla$ wangyanwang@bjmu.edu.cn )

Peking University First Hospital https://orcid.org/0000-0002-8577-0527

\section{Guiqiang Wang}

Peking University First Hospital

\section{Research article}

Keywords: liver stiffness measurements, shear wave elastography, transient elastography, dependent factor, AUROC, chronic hepatitis B

Posted Date: December 4th, 2019

DOI: https://doi.org/10.21203/rs.2.18042/v1

License: (c) (1) This work is licensed under a Creative Commons Attribution 4.0 International License. Read Full License 


\section{Abstract}

Background Noninvasive measurements including transient elastography (TE) and the two-dimensional shear wave elastography (SWE) have been clinical used instead of liver biopsy for the regular assessment of liver fibrosis in patients during following-up. We aimed to investigate independent factors of SWE compared with TE and find the optimal cutoff values of these two non-invasive evaluation assessments based on our cohort of treatment-naive CHB patients.

Methods Fifty-four treatment-naive CHB patients were enrolled and studied. All of them were performed SWE, TE, serum tests and liver biopsy. Potential factors of SWE and TE values were analysed by linear regression and orthogonal partial least squares (OPLS) discriminant analysis. The agreement, the association, the comparison of the two different methods were implemented based on the results of liver biopsy by using Bland-Altman analysis, spearman's correlation and areas under the receive operating characteristic curves (AUROCs).

Results There were 27 cases (50\%) of mild fibrosis (F0-F2) and 27 (50\%) cases of significant fibrosis (F3F6), fibrosis was assessed with the Ishak scoring system. Multivariate linear regression analyses revealed that fibrosis stage was the only factor affecting the values of SWE $(P<0.001)$, whereas total bilirubin levels $(P=0.013)$ and fibrosis stage $(P=0.037)$ were independent factors affecting TE values. OPLS showed the numbers independent factors $(\mathrm{VIP}>1)$ were more in TE than SWE. Bland-Altman analysis showed satisfied agreement between LSMs of SWE and TE. Both of SWE and TE could significantly evaluate significant fibrosis $(P<0.001)$. Spearman's correlation analysis revealed a correlation between liver fibrosis and LSMs of SWE and TE $(r=0.65$ and $0.50, P<0.001)$. Areas under receiver operating characteristics curves (AUROCs) of SWE and TE for significant fibrosis ( $F>2$ ) were 0.786 and 0.714 , respectively. The optimal cutoff values of LSMs of SWE and TE were $9.05 \mathrm{kPa}$ and $8.15 \mathrm{kPa}$, respectively.

Conclusions According to the AUROCs in comparative data, SWE may be more sensitive and more precise than TE in predicting significant fibrosis ( $>F 2$ in $\mathrm{CHB}$ patients. Compare to TE, the value of SWE is less affected by factors and will be more promising application prospects.

\section{Background}

Assessments of liver fibrosis in chronic hepatitis B (CHB) patients is crucial since there are different antiviral therapy strategies in CHB patients without or with significant-to-severe liver fibrosis and it is the regular assessment in following-up patients. Evaluating the degree of liver fibrosis is cornerstone before treatment in treatment-naïve CHB patients [1]. Liver biopsy is still considered the golden standard to evaluate liver fibrosis nowadays [2]. However, the complications after Liver Biopsy [3], sampling error as well as the disability of monitor during follow-up period associated with the invasive operation, result in noninvasive methods of liver stiffness measurement (LSM) rather than liver biopsy are more likely to be chosen as a dynamic monitoring item during the whole therapy and following-up period in CHB patients. 
Transient elastography (TE) is a non-invasive, valid, rapid, reproducible and widely-used method for evaluating liver stiffness by measuring the velocity of elastic shear waves in the liver parenchyma [4]. However, the value of TE is unreliable in some conditions, such as high alanine aminotransferase levels, overweight or thick abdominal fat, hepatic inflammatory activity, extrahepatic cholestasis, ascites, older even a narrow intercostal space [5-7].Two-dimensional shear wave elastography (SWE) is another noninvasive LSM assessment method which is installed on the traditional ultrasound machine. Compared with TE, SWE can be conveniently performed using a conventional ultrasound scanner and create a realtime, 2-D map of liver tissue stiffness under the guidance of B-mode imaging. Now, SWE has proven to be one of reliable methods of liver stiffness for chronic liver diseases [8-12]. There are also few studies about SWE in the assessment of liver fibrosis with histologic confirmation for treatment-naïve CHB patients compared with other non-invasive methods [13-15]. But there have been no published studies evaluating independent factor of SWE compared with TE in patients with CHB infection. Therefore, the aim of this study was to analysis the potential factors of SWE and TE in CHB patients and find the optimal cutoff values of these two non-invasive evaluation assessments based on our cohort of treatment-naive CHB patients.

\section{Methods}

Patients: Between October 2013 and May 2015, 54 patients with CHB infection who underwent liver biopsy to assess liver fibrosis were prospectively considered for inclusion in this study. Written informed consents were obtained from all the patients, and the study was approved by the Ethics Committee of Peking University First Hospital. CHB infection was diagnosed when hepatitis B surface antigen (HBsAg) were present in the serum for at least 6 months, the patients with decompensate liver cirrhosis and hepatocarcinoma (HCC) were not enrolled. Other exclusion criteria were: hepatitis $\mathrm{C}$ virus (HCV) or human immunodeficiency virus (HIV) coinfection; other chronic liver diseases such as alcoholic, nonalcoholic fatty, autoimmune, drug-induced and genetic; age $<18$ year; pregnancy women; and patients who failed to complete SWE or TE. Clinical data, including basic information (age, gender, weight, height), blood test results and LSMs, were recorded one month before or after liver biopsy. The blood tests include: WBC, white blood cell count; NEU, neutrophils count; HB, hemoglobin; PLT, platelet count; ALT, alanine aminotransferase; AST, aspartate aminotransferase; TBIL, total bilirubin; $\mathrm{CHE}$, cholinesterase; SCR, serum creatinine; PT, prothrombin time; APTT, activated partial thromboplastin time; PTA, prothrombin activity; INR, international normalized ratio; $\mathrm{HBsAb}$, hepatitis B surface antibody; $\mathrm{HBeAg}$, hepatitis B e antigen; $\mathrm{HBeAb}$, hepatitis $\mathrm{B}$ e antibody. Body mass index (BMI) was calculated as the weight $(\mathrm{kg}) /(\text { height }[\mathrm{m}])^{2}$.

Liver histological assessment: All patients performed liver biopsies in the guide of ultrasonography. A minimum of $20 \mathrm{~mm}$ of liver biopsy with at least 11 portal tracts was considered valid for diagnosis. All liver biopsies were blindly and independently reviewed by two hepato-pathologists from Beijing You An Hospital affiliated to Capital Medical University. When discrepancies occurred, final decision was made by the third experienced hepato-pathologist who was also responsible for reassessment of $10 \%$ samples by random drawing. Inflammation grade and fibrosis stage were assessed with the Ishak scoring system 
[16]. Fibrosis was scored as follows: $F$ 0-1, no/mild fibrosis; $F \geq 2$, moderate fibrosis; $F \geq 3$, significant fibrosis; $F \geq 4$, advanced fibrosis; and $F \geq 5$, cirrhosis. Inflammation grading was performed using the modified histology activity index (HAl) and scored as follows: HAI 0-4, no/mild inflammation; HAI 5-6, moderate inflammation; $\mathrm{HAI} \geq 7$, severe inflammation.

TE: TE was performed on fasting patients using one-dimensional ultrasound TE (FibroScan, Echosens, Paris, France) with an M probe. The operator had experience with at least $100 \mathrm{TE}$ procedures and she was blind of the patients' clinical data. Liver stiffness measurements (LSMs) were obtained from the right lobe of the liver through the intercostal spaces with the patient lying in the dorsal decubitus position and the right arm in maximal abduction. The operator pressed the probe button then to begin the measurements. The unit of LSM values are expressed in kilopascal $(\mathrm{kPa})$. Reliable measurements were considered with at least 10 valid measurements, an interquartile range/median (IQR) value $<30 \%$ and a success rate $>60 \%[4]$.

SWE: Two-dimensional SWE was also performed on fasting patients using the Aixplorer US system (SuperSonic Imagine, Aix-en-Provence, France) with a convex broadband probe (SC6-1, 1-6 MHz). A radiologist (X. L. Tian) who had more than 30 years of experience in performing ultrasonic performed the procedures. She was also blinded to the patients' clinical data and TE results. The operator lay in the supine position and his/her right arm were in maximal abduction then 2D SWE measurements were performed on the right lobe of the liver through the intercostal spaces. When the target area was located, SWE was launched, and the patient was asked to hold breath during quiet breathing for approximately 5 s. The elasticity image box, which was approximately $4 \times 3 \mathrm{~cm}$, was in an area of the liver parenchyma free of large vessels and bile ducts. Then a circular region of interest (ROI) with a $2 \mathrm{~cm}$ diameter was positioned in an area of homogeneous color, the liver stiffness means, minimum, maximum, and standard deviation (SD) were calculated automatically. The mean value was used in the analysis to represent the LSMs. Measurements were considered failures when little or no signal was obtained.

Statistical analyses: Descriptive statistics were summarized as mean \pm standard deviation (SD) or median \pm interquartile range (IQR). Differences between SWE and TE were recorded as Bland-Altman plot to evaluate the agreement. Comparisons between groups were made with the non-parametric test for quantitative variables. Correlations between noninvasive methods and liver fibrosis stages were identified with the Spearman's correlation test. Factors affecting liver stiffness values of SWE and TE were first analyzed with univariate linear testing, and those with $P<0.05$ were subsequently included in a multivariate linear regression analysis. Orthogonal partial least squares discriminant analysis (OPLS-DA) was used to rank the ability of the parameters to affect the LSMs of SWE and TE using SIMCA software (version 14.0; Umetrics AB, Umea, Sweden). Performance of noninvasive methods in the assessment of liver fibrosis stages was determined by using receiver operating characteristic (ROC) curves. Areas under the receiver operating characteristic curve (AUROCs) were used to estimate the probability of the correct prediction of liver fibrosis stages. Differences between various AUROCs were compared by using a Delong test. (Sensitivity+specificity-1) was for cutoff value. Positive and negative predictive values, positive and negative diagnostic likelihood ratios were calculated based on for the cutoff values. All 
statistical tests were two sided, and $\mathrm{P}$ values less than 0.05 indicated statistical significance. The statistical analyses were performed using SPSS 23.0 (SPSS Inc., Chicago, IL, USA) and Medcalc (Version 19.0.7; MedCalc Software bvba, Mariakerke, Belgium).

\section{Results}

\section{Patient characteristics}

Fifty-four Chinese treatment-naïve CHB patients were eligible for the study who is successful for SWE and TE (Table 1). Ishak HAI grades, liver fibrosis scores, LSMs were all available. All 54 patients were antiviral therapy-naive CHB patients which included 41 men and 13 women, with a mean age of 36.7 years. The medium BMI was $23.9 \mathrm{~kg} / \mathrm{m}^{2}$. The levels of serum ALT were shown in medium and interquartile range with a higher level than the reports (Additional file 1). The medium of total bilirubin was $14.5 \mu \mathrm{mol} / \mathrm{L}$, rang from $7.2 \mu \mathrm{mol} / \mathrm{L}$ to $45.0 \mu \mathrm{mol} / \mathrm{L}$, which was as same as the reports (Additional file 1 ). The mean $\log _{10} \mathrm{HBV}$ DNA was 4.8 , the mean HBV-DNA levels was $8.8 \times 10^{4} \mathrm{IU} / \mathrm{ml}$. Almost half of the population were diagnosed with no/mild fibrosis stage and the other half were diagnosed with $\mathrm{F}>2$ which mean significant fibrosis, histopathologically. About $80 \%$ of the population were significant-severe inflammatory. Basic demographic, clinical and laboratory characters of these patients were summarized in Table 1 and the table of population characteristics compared with previous reports were shown in additional file 1.

\section{Analyses of independent parameters associated with LSMS of SWE and TE}

We investigated the factors that affect liver stiffness values by SWE and TE. The parameters included gender, age, BMI, inflammatory grade (HAl), liver fibrosis stage, WBC count, Neutrophil count, HB, PLT count, ALT, AST, TBIL, CHE, Scr, PT, APTT, PTA, INR, HBsAb, HBeAg, HBeAb and $\lg _{10} \mathrm{HBV}$ DNA (Table 1). Concerning SWE, a univariate linear analysis revealed corrections between LSMs of SWE and liver fibrosis stage, TBIL, $\lg _{10} \mathrm{HBV}$ DNA and portal vein diameter (PVD), while a multivariate analysis showed that only the liver fibrosis stage was an independent factor affecting LSMs of SWE $(P<0.05)$. Concerning TE, a univariate analysis revealed correlations between LSMs of TE and liver fibrosis stage, PLT and TBIL, while a multivariate analysis showed that not only the liver fibrosis stage but also TBIL was independent factors affecting LSMs of TE $(P<0.05)$.

Orthogonal partial least squares (OPLS) discriminant analysis was further used to rank the affections of the parameters to SWE and TE. As shown in figure 1, the top predictors were liver fibrosis for both SWE (Figure 1A) and TE (Figure 1B), while higher VIP predictors $(>1)$ were much more in TE than SWE. This mean the value of SWE is less effected by factors and is a reliable method which would be more broad clinical application prospects. 


\section{Agreement and association between SWE, TE and histological hepatitis fibrosis}

Bland-Altman analysis is used to assess the agreement between two measurements. It revealed that the mean difference of the two measurements was $1.3 \mathrm{kPa}$, the standard deviation (SD) is $3.0 \mathrm{kPa}$, and the upper and lower limits of the mean difference $(95 \% \mathrm{Cl}$ ) were $11.2 \mathrm{kPa}$ and $-8.7 \mathrm{kPa}$, respectively (Figure 2). It showed satisfied agreement of LSMs between SWE and TE.

All 54 patients had been given a confirmed histologic diagnosis by using Ishak scores as the reference methods. The measurements of SWE and TE for different fibrosis stages are

shown in Table 2. Increasing liver stiffness values were highly correlation with progressing stage of fibrosis. The LSM increased as the fibrosis stage elevated with both SWE and TE $(P<0.001$, Figure 3$)$, while TE showed a wide range of larger SDs compared with SWE.

Spearman's correlation between different noninvasive approaches were also analyzed. The association was stronger between fibrosis stages and SWE which shown a moderate correlation $(r=0.65, P<0.001)$, compared with the associations between fibrosis stages and TE which shown a weak correlation $(r=$ $0.50, P<0.001)$. LSMs of SWE and TE with the different liver fibrosis stages in comparison are shown in Figure 3. SWE and TE shown statistical significance in distinguishing mild liver fibrosis and significant liver fibrosis.

\section{Comparison of performance in SWE and TE to diagnose significant liver fibrosis stages}

The areas under ROC curve (AUCs), cut-off values, sensitivity, specificity, positive

predictive values, and negative predictive values for the diagnosis of mild liver fibrosis $(F \leq 2)$ and significant liver fibrosis $(F>2)$ using SWE and TE are shown in Table 3. For predicting significant fibrosis (>F2, Figure 4), AUROCs of LSMs were 0.786 for SWE (95\% Cl $0.661-0.911)$ and 0.714 for TE $(95 \% \mathrm{Cl}$ 0.573-0.855) (Figure 4). There were no statistically significant differences in the AUROCs between SWE and TE for significant fibrosis ( $P>0.05$ ). Optimal cutoff values of $L S M s$ for significant fibrosis were 9.05 $\mathrm{kPa}$ for SWE and $8.15 \mathrm{kPa}$ for TE, respectively (Table 3).

\section{Discussion}

Early diagnosis of significant liver fibrosis is particularly important for clinical treatment decision in the treatment-naïve CHB patients, especially in the patients with normal ALT and mildly elevated ALT (<2ULN) who were considered "inactive" [1]. For this consideration, 55\% (24/44) patients with normal or mildly elevated ALT analyzed in our study should initiate antiviral therapy because they were diagnosed with significant fibrosis histopathologically. However, it is hard to perform regularly liver biopsy during follow 
up period for evaluation of antiviral treatment effect. Therefore, finding noninvasive methods for diagnosis of significant fibrosis is urgent, and it can reduce the need of liver biopsy and assist making treatment decisions.

SWE is a relatively new technique that is based on shear waves implemented on a diagnostic ultrasound system and estimates the speed of a shear wave to provide a quantitative estimate of tissue stiffness like TE [17]. With consideration of interference factors which may affect the reliable of LSMs, we analysis the regression of the SWE with TE. The result of our study shown liver fibrosis stage was the only independent factor affecting the LSMs of SWE, whereas TBIL as well as liver fibrosis stage can affect the LSMs of TE. And this result was consistent with previous study of TE $[5,7]$, while is contrary from one published study of SWE [18]. OPLS showed the most independent factor was fibrosis stage for both SWE and TE, while the numbers independent factors (VIP>1) were more in TE than SWE. In Zhuang Y's study, the LSMs of SWE was affected not only by liver fibrosis stage, variables such as inflammation and AST, ALT, and glutamyl transpeptidase (GGT) levels influenced LSMs [18]. Another analysis of Jie Zeng also analysis that aminotransferase levels influence LSMs of SWE in index cohort and TBIL levels influence in validation cohort [13]. In our present study, the value of SWE was not significantly associated with the above factors reported, it may because the cohort of the present study is consisting of CHB people whose aminotransferase are higher than the reported SWE results (Additional file 1). The higher aminotransferase result in that no factor was selected in SWE. To investigate whether different levels of inflammatory grade affected the performances of SWE and TE, stratification analysis in subgroups was performed. The results revealed that neither for $\mathrm{F} \leq 2$ or $\mathrm{F}>2$, the inflammation grade didn't show significant impact on the performance of SWE or TE. This result is inconsistent with previous reports [18], it may be caused by the higher inflammatory grade of our study population, whose proportion of moderate to severe inflammation was $80 \%$ (Table 1). Further large-scale research is needed to verify if severe inflammatory response in liver could impact on SLMs measurement.

The comparison between the liver elasticity values assessed by SWE and TE showed a good measurement with a mean difference value of $1.3 \mathrm{kPa}$. It indicated that the measurement results of the two methods have good consistency. Our results showed that both SWE and TE were able to discriminate significant fibrosis from normal or mild fibrosis, whereas TE showed a broad range. Comparing with TE, SWE showed a higher correlation coefficient value with fibrosis stage $(r=0.65$ and 0.50$)$.

We further evaluated the validation of SWE and TE and the diagnostic accuracy of the two methods in patients with CHB patients. The AUROCs were numerically slightly higher for SWE than for TE, despite the absence of statistically significant differences, SWE had a higher accuracy which sensitivity and specificity is higher than TE. The findings of our study indicated that SWE has better diagnostic accuracy in the assessment of significant liver fibrosis in patients with CHB infection, though both two methods could make an accuracy assessment between mild fibrosis and significant fibrosis based on histologic diagnosis. The possible assumption is that SWE has the advantage of real-time 2-D imaging under the guidance of B-mode imaging, allowing this method to yield a circular ROI positioned in a homogeneous area of the liver stiffness, which could avoid large blood vessels and other tissues in liver. While 
comparing with SWE, the probe location of TE is the only factor determined by the operator. The results of our study are similar to a previous study on the elastography assessment of liver fibrosis comparing SWE and TE of CHB patients $[14,15]$. The cut-off value of SWE for identifying significant fibrosis $(F>2)$ in our treatment-naive CHB patients showed $9.05 \mathrm{kPa}$, which is higher than the reported study [13-15], while the mean liver stiffness determined with SWE in the cohort of participants with healthy livers was $5.1 \pm 1.3$ $\mathrm{kPa}$ [19]. The cut-off of TE values for identifying significant fibrosis in our CHB patients showed $8.15 \mathrm{kPa}$, which is also higher than the reported study [15]. The higher cutoff values of our cohort may due to the elevated transaminase and severe inflammatory than the reported studies (Additional file 1) $[7,19]$. The patients of our study are the distinguished group of people with active hepatitis B which is supported by liver biopsy HAI scores rather than a cohort mixed with hepatitis B carriers or inactive CHB patients. It may provide evidence for making therapeutic decision.

The advantage of this study is fifty-four treatment-naive CHB patients were enrolled and studied prospectively in our study. All of patients underwent liver biopsy, the samples of liver biopsy meet the American Society for the Study of Liver Diseases criteria of a sample of at least 2-3 cm in length and with at least 11 portal tracts [3]. Biopsy specimens were analyzed by experienced liver pathologists. The assessment of the inter-observer variability was controlled by the third experienced liver pathologist. Besides that, in consideration of the data of SWE and TE may have bias between different operators, we designated one operator for SWE and TE. Based on the reliable data, we analysis the potential factors affect the measurement of SWE and TE and reveal that vividly in figures. Furthermore, we confirmed SWE and TE are both excellent assessments to predict liver fibrosis in CHB patients. According to the AUROCs in comparative data, SWE may be more sensitive and more precise than TE in predicting significant fibrosis ( $>\mathrm{F} 2)$ in $\mathrm{CHB}$ patients. The value of SWE is more reliable and less affected by various factors compare to TE. It might help identify CHB patients who may benefit from treatment. Last, the limitation of the study is that the sample size is relatively small, the present results need to be verified in further largescale trials.

\section{Conclusions}

These findings suggest that LSMs by SWE and TE were strongly correlated with the results of liver biopsy, which indicated SWE and TE are good diagnostic performance in assessing liver fibrosis stages. SWE can be successfully used for the assessment of liver fibrosis stages in patients with CHB. Since SWE provides comparable diagnostic accuracy without affection of various factors, it would be more broad clinical application prospects on standard examinations routinely in HBV patients.

\section{List Of Abbreviations}

ALB, albumin; ALT, alanine aminotransferase; APTT, activated partial thromboplastin time; AST, aspartate aminotransferase; AUROC: areas under the receive operating characteristic curve; BMI, body mass index; CHB: chronic hepatitis $\mathrm{B}$; $\mathrm{CHE}$, cholinesterase; GGT, gamma-glutamyl transpeptidase; HAl: histology activity index; HB, hemoglobin; HBeAb, hepatitis B e antibody; HBeAg, hepatitis B e antigen; HBsAb, 
hepatitis B surface antibody; HBsAg: hepatitis B surface antigen; HBV-DNA: hepatitis B virus DNA; HCC: hepatocarcinoma; INR, international normalized ratio; IQR: interquartile range; LR-, negative likelihood value; $L R+$, positive likelihood value; LSM: liver stiffness measurement; NEU, neutrophils; NPV, negative predictive value; PLT, platelet; PPV, positive predictive value; PT, prothrombin time; PTA, prothrombin activity; PVD, portal vein diameter; SCR, serum creatinine; SD: standard deviation; ST, spleen thickness; SWE: shear wave elastography; TBIL, total bilirubin; TE, transient elastography; WBC, white blood cell.

\section{Declarations}

\section{Ethics approval and consent to participate}

The study was approved by the Ethics Committee of Peking University First Hospital and conformed to the guidelines set forth by the 1964 Helsinki.

\section{Consent for publication}

Not applicable.

\section{Availability of data and materials}

The datasets used and/or analysed during the current study are available from the corresponding author on reasonable request.

\section{Competing interests}

The authors declare that they have no competing interests.

\section{Funding}

This work was supported in part by the National Natural Science Foundation of China (81870417 to YW) and sub-subject of the major projects of national science and technolgy (2018ZX10302206-001-007 to YW; 2017ZX10203202-002-012 to YW; 2017ZX10302201-003-003 to QW).

\section{Authors' contributions}

TY collected, analysed and interpreted data and contributed in writing the manuscript. JP collected and analysed data. DQ and HC contributed for the statistic work. YW contributed to discussion and manuscript revision. YW and QW designed, drafted the work and substantively revised it, they contributed to this work equally. All authors read and approved the final manuscript. 


\section{Acknowledgements}

We sincerely thank Dr. X. L. Tian and Dr S. N. Zhu's contributions for this study.

\section{References}

1.Terrault NA, Lok ASF, McMahon BJ, Chang KM, Hwang JP, Jonas MM, Brown RS, Jr., Bzowej NH, Wong JB: Update on prevention, diagnosis, and treatment of chronic hepatitis B: AASLD 2018 hepatitis B guidance. Hepatolgy 2018, 67(4):1560-1599.

2.Bravo AA, Sheth SG, Chopra S: Liver biopsy. N Engl J Med 2001, 344(7):495-500.

3.Rockey DC, Caldwell SH, Goodman ZD, Nelson RC, Smith AD: Liver Biopsy. Hepatolgy 2009, 49(3):1017-1044.

4.Sandrin L, Fourquet B, Hasquenoph JM, Yon S, Fournier C, Mal F, Christidis C, Ziol M, Poulet B, Kazemi F et al: Transient elastography: a new noninvasive method for assessment of hepatic fibrosis. Ultrasound Med Biol 2003, 29(12):1705-1713.

5.Trifan A, Sfarti C, Cojocariu C, Dimache M, Cretu M, Hutanasu C, Stanciu C: Increased liver stiffness in extrahepatic cholestasis caused by choledocholithiasis. Hepat Mon 2011, 11(5):372-375.

6.Yu JH, Lee Jl: Current role of transient elastography in the management of chronic hepatitis B patients. Ultrasonography 2017, 36(2):86-94.

7.Arena U, Vizzutti F, Corti G, Ambu S, Stasi C, Bresci S, Moscarella S, Boddi V, Petrarca A, Laffi G et al: Acute viral hepatitis increases liver stiffness values measured by transient elastography. Hepatolgy 2008 , 47(2):380-384.

8.Mjelle AB, Mulabecirovic A, Hausken T, Havre RF, Gilja OH, Vesterhus M: Ultrasound and Point Shear Wave Elastography in Livers of Patients with Primary Sclerosing Cholangitis. Ultrasound Med Biol 2016, 42(9):2146-2155.

9.Cassinotto C, Lapuyade B, Mouries A, Hiriart JB, Vergniol J, Gaye D, Castain C, Le Bail B, Chermak F, Foucher $\mathrm{J}$ et al: Non-invasive assessment of liver fibrosis with impulse elastography: comparison of Supersonic Shear Imaging with ARFI and FibroScan(R). J Hepatol 2014, 61(3):550-557.

10.Ferraioli G, Tinelli C, Dal Bello B, Zicchetti M, Filice G, Filice C, Liver Fibrosis Study G: Accuracy of realtime shear wave elastography for assessing liver fibrosis in chronic hepatitis C: a pilot study. Hepatolgy 2012, 56(6):2125-2133.

11.Herrmann E, de Ledinghen V, Cassinotto C, Chu WCW, Leung VYF, Ferraioli G, Filice C, Castera L, Vilgrain V, Ronot $\mathrm{M}$ et al: Assessment of biopsy-proven liver fibrosis by two-dimensional shear wave elastography: An individual patient data-based meta-analysis. Hepatolgy 2018, 67(1):260-272. 
12.Takeuchi H, Sugimoto K, Oshiro H, Iwatsuka K, Kono S, Yoshimasu Y, Kasai Y, Furuichi Y, Sakamaki K, Itoi T: Liver fibrosis: noninvasive assessment using supersonic shear imaging and FIB4 index in patients with non-alcoholic fatty liver disease. J Med Ultrason (2001) 2018, 45(2):243-249.

13.Zeng J, Liu GJ, Huang ZP, Zheng J, Wu T, Zheng RQ, Lu MD: Diagnostic accuracy of two-dimensional shear wave elastography for the non-invasive staging of hepatic fibrosis in chronic hepatitis $\mathrm{B}$ : a cohort study with internal validation. Eur Radiol 2014, 24(10):2572-2581.

14.Wu T, Wang P, Zhang T, Zheng J, Li S, Zeng J, Kudo M, Zheng R: Comparison of Two-Dimensional Shear Wave Elastography and Real-Time Tissue Elastography for Assessing Liver Fibrosis in Chronic Hepatitis B. Dig Dis 2016, 34(6):640-649.

15.Zeng J, Zheng J, Huang Z, Chen S, Liu J, Wu T, Zheng R, Lu M: Comparison of 2-D Shear Wave Elastography and Transient Elastography for Assessing Liver Fibrosis in Chronic Hepatitis B. Ultrasound Med Biol 2017, 43(8):1563-1570.

16.Ishak K, Baptista A, Bianchi L, Callea F, De Groote J, Gudat F, Denk H, Desmet V, Korb G, MacSween RN et al: Histological grading and staging of chronic hepatitis. J Hepatol 1995, 22(6):696-699.

17.Nadebaum DP, Nicoll AJ, Sood S, Gorelik A, Gibson RN: Variability of Liver Shear Wave Measurements Using a New Ultrasound Elastographic Technique. J Ultrasound Med 2018, 37(3):647-656.

18.Zhuang Y, Ding H, Zhang Y, Sun HC, Xu C, Wang WP: Two-dimensional Shear-Wave Elastography Performance in the Noninvasive Evaluation of Liver Fibrosis in Patients with Chronic Hepatitis B:

Comparison with Serum Fibrosis Indexes. Radiolgy 2017, 283(3):872-881.

19.Bende F, Mulabecirovic A, Sporea I, Popescu A, Sirli R, Gilja OH, Vesterhus M, Havre RF: Assessing Liver Stiffness by 2-D Shear Wave Elastography in a Healthy Cohort. Ultrasound in Medicine and Biolgy 2018, 44(2):332-341.

\section{Tables}

Table 1. Basic demographic, laboratory characters of the study population and factors associated with the LSMs measured by SWE and TE in univariate and multivariate linear regression analyses. 


\begin{tabular}{|c|c|c|c|c|c|}
\hline & Variables & SWE (P val & & TE (P value & \\
\hline & & $\begin{array}{l}\text { Univariate } \\
\text { analysis }\end{array}$ & $\begin{array}{l}\text { Multivariate } \\
\text { analysis }\end{array}$ & $\begin{array}{l}\text { Univariate } \\
\text { analysis }\end{array}$ & $\begin{array}{l}\text { Multivariate } \\
\text { analysis }\end{array}$ \\
\hline $\begin{array}{l}\text { Gender } \\
(\mathrm{M} / \mathrm{F})\end{array}$ & $41(76 \%) / 13(24 \%)$ & 0.217 & & 0.072 & \\
\hline Age (y) & $36.7,9.96,19.0-61.0^{*}$ & 0.823 & & 0.509 & \\
\hline $\begin{array}{l}\mathrm{BMI} \\
\left(\mathrm{kg} / \mathrm{m}^{2}\right)\end{array}$ & $\begin{array}{l}23.9(21.9-25.0)[16.4- \\
30.3] \S\end{array}$ & 0.818 & & 0.232 & \\
\hline HAI & $\begin{array}{ll}\text { HAI 0-4 } & 11(20.4 \%) \\
\text { HAI 5-6 } & 18(33.3 \%)\end{array}$ & 0.659 & & 0.184 & \\
\hline Fibrosis & $\begin{array}{ll}\text { HAI 7-12 } & 25(46.3 \%) \\
\text { F0 } & 3(5.6 \%) \\
\text { F1 } & 4(7.4 \%) \\
\text { F2 } & 20(37.0 \%) \\
\text { F3 } & 22(40.7 \%) \\
\geq \text { F4 } & 5(9.3 \%)\end{array}$ & 0.000 & 0.000 & 0.000 & 0.037 \\
\hline $\begin{array}{l}\text { WBC } \\
\left(10^{9} / \mathrm{L}\right)\end{array}$ & $5.5(4.3-6.6)[1.7-9.4] \S$ & 0.078 & & 0.173 & \\
\hline $\begin{array}{l}\mathrm{NEU} \\
\left(10^{9} / \mathrm{L}\right)\end{array}$ & $3.1(2.3-3.9)[1.0-7.5] \S$ & 0.490 & & 0.500 & \\
\hline $\begin{array}{l}\mathrm{HB} \\
\left(10^{9} / \mathrm{L}\right)\end{array}$ & $\begin{array}{l}151.0(139-165)[100.0- \\
180.0] \S\end{array}$ & 0.053 & & 0.184 & \\
\hline $\begin{array}{l}\text { PLT } \\
\left(10^{9} / \mathrm{L}\right)\end{array}$ & $\begin{array}{l}\text { 166.5(128.3-219.8) } \\
{[65.0-327.0] \S}\end{array}$ & 0.088 & & 0.006 & 0.089 \\
\hline ALT (IU/L) & $\begin{array}{l}50.4(28.8-129.2)[12.5- \\
402.9] \S\end{array}$ & 0.737 & & 0.059 & \\
\hline $\begin{array}{l}\text { AST } \\
\text { (IU/L) }\end{array}$ & $\begin{array}{l}38.9(26.5-67.0)[16.1- \\
179.2] \S\end{array}$ & 0.338 & & 0.531 & \\
\hline $\begin{array}{l}\text { TBIL } \\
(\mu \mathrm{mol} / \mathrm{L})\end{array}$ & $\begin{array}{l}14.5(11.9-20.7)[7.2- \\
45.0] \S\end{array}$ & 0.046 & 0.512 & 0.000 & 0.013 \\
\hline $\begin{array}{l}\mathrm{CHE} \\
(\mathrm{IU} / \mathrm{L})\end{array}$ & $\begin{array}{l}7635(6335-9076) \\
{[2138-16444] \S}\end{array}$ & 0.925 & & 0.191 & \\
\hline $\begin{array}{l}\text { SCR } \\
(\mu \mathrm{mol} / \mathrm{L})\end{array}$ & $\begin{array}{l}70.1(55.7-81.5)[40.0- \\
120.2] \S\end{array}$ & 0.719 & & 0.916 & \\
\hline $\mathrm{PT}(\mathrm{s})$ & $\begin{array}{l}11(10.5-11.8)[9.2- \\
13.7] \S\end{array}$ & 0.879 & & 0.125 & \\
\hline APTT (s) & $\begin{array}{l}14.5(31.9-36.3)[0.9- \\
43.1] \S\end{array}$ & 0.754 & & 0.587 & \\
\hline PTA (\%) & $\begin{array}{l}101.0(90.0-111.5)[69.0- \\
122.0] \S\end{array}$ & 0.612 & & 0.091 & \\
\hline INR & $1.03(0.9-1.1)[0.9-1.6] \S$ & 0.106 & & 0.475 & \\
\hline $\begin{array}{l}\text { HBsAb } \\
(-/+)\end{array}$ & $46(85 \%) / 7(13 \%)$ & 0.270 & & 0.167 & \\
\hline $\begin{array}{l}\text { HBeAg } \\
(-/+)\end{array}$ & $21(39 \%) / 33(61 \%)$ & 0.772 & & 0.405 & \\
\hline $\begin{array}{l}\mathrm{HBeAb} \\
(-/+)\end{array}$ & $27(50 \%) / 27(50 \%)$ & 0.539 & & 0.523 & \\
\hline $\begin{array}{l}\log _{10} \mathrm{HBV} \\
\text { DNA }\end{array}$ & $4.8(2.7-6.3)[1.3-8.4] \S$ & 0.045 & 0.148 & 0.711 & \\
\hline
\end{tabular}

*Mean, SD, range.

- Median (interquartile range) [range].

Abbreviation: BMI, body mass index; HAI, histology activity index; WBC, white blood cell count; NEU, neutrophils count; HB, hemoglobin; PLT, platelet count; ALT, alanine aminotransferase; AST, aspartate 
aminotransferase; TBIL, total bilirubin; CHE, cholinesterase; SCR, serum creatinine; PT, prothrombin time; APTT, activated partial thromboplastin time; PTA, prothrombin activity; INR, international normalized ratio; HBsAb, hepatitis B surface antibody; HBeAg, hepatitis B e antigen; HBeAb, hepatitis B e antibody.

Table 2. Statistic of LSMs by SWE and TE with Spearman's coefficients according to liver fibrosis.

\begin{tabular}{|c|c|c|c|c|c|c|}
\hline & \multicolumn{2}{|c|}{$\mathrm{F} \leq 2(\mathrm{n}=27)$} & \multicolumn{2}{|c|}{$\mathrm{F}>2(\mathrm{n}=27)$} & \multirow[t]{2}{*}{$\mathrm{r}$} & \multirow[t]{2}{*}{ P value } \\
\hline & F0-1 $(n=7)$ & $\mathrm{F} 2(\mathrm{n}=20)$ & $F 3(n=22)$ & F4-6 $(\mathrm{n}=5)$ & & \\
\hline SWE (Mean \pm SD) & $\begin{array}{l}6.6 \pm 1.7 \\
8.0 \pm 3.0\end{array}$ & $7.6 \pm 3.2$ & $\begin{array}{l}10.2 \pm 2.8 \\
12.1 \pm 5.3\end{array}$ & $20.5 \pm 6.0$ & 0.65 & $<0.001$ \\
\hline TE $($ Mean \pm SD $)$ & $\begin{array}{l}6.8 \pm 2.1 \\
9.3 \pm 6.1\end{array}$ & $8.1 \pm 6.8$ & $\begin{array}{l}11.1 \pm 4.7 \\
13.3 \pm 7.0\end{array}$ & $23.5 \pm 6.9$ & 0.50 & $<0.001$ \\
\hline
\end{tabular}

Table 3. Performance characteristics of SWE and TE for staging liver fibrosis in CHB patients. PPV (NPV), positive (negative) predictive value; LR+ (LR-), positive (negative) likelihood value.

\begin{tabular}{lllllllll}
\hline Parameters & AUROC & $\begin{array}{l}\text { Cutoff Value } \\
(\mathrm{kPa})\end{array}$ & $\begin{array}{l}\text { Sensitivity, } \\
\%\end{array}$ & $\begin{array}{l}\text { Specificity, } \\
\%\end{array}$ & $\begin{array}{l}\text { PPV, } \\
\%\end{array}$ & $\begin{array}{l}\text { NPV, } \\
\%\end{array}$ & LR+ & LR- \\
\hline SWE & 0.786 & 9.05 & 77.78 & 77.78 & 77.78 & 77.78 & 3.50 & 0.29 \\
& & & & & & & & \\
TE & 0.714 & 8.15 & 59.26 & 66.67 & 64.00 & 62.07 & 1.78 & 0.61 \\
\hline
\end{tabular}

Abbreviation: PPV (NPV), positive (negative) predictive value; LR+ (LR-), positive (negative) likelihood value. Characteristics are based on optimal cutoff elasticity values. Pathologic analysis was the diagnostic reference standard.

\section{Figures}



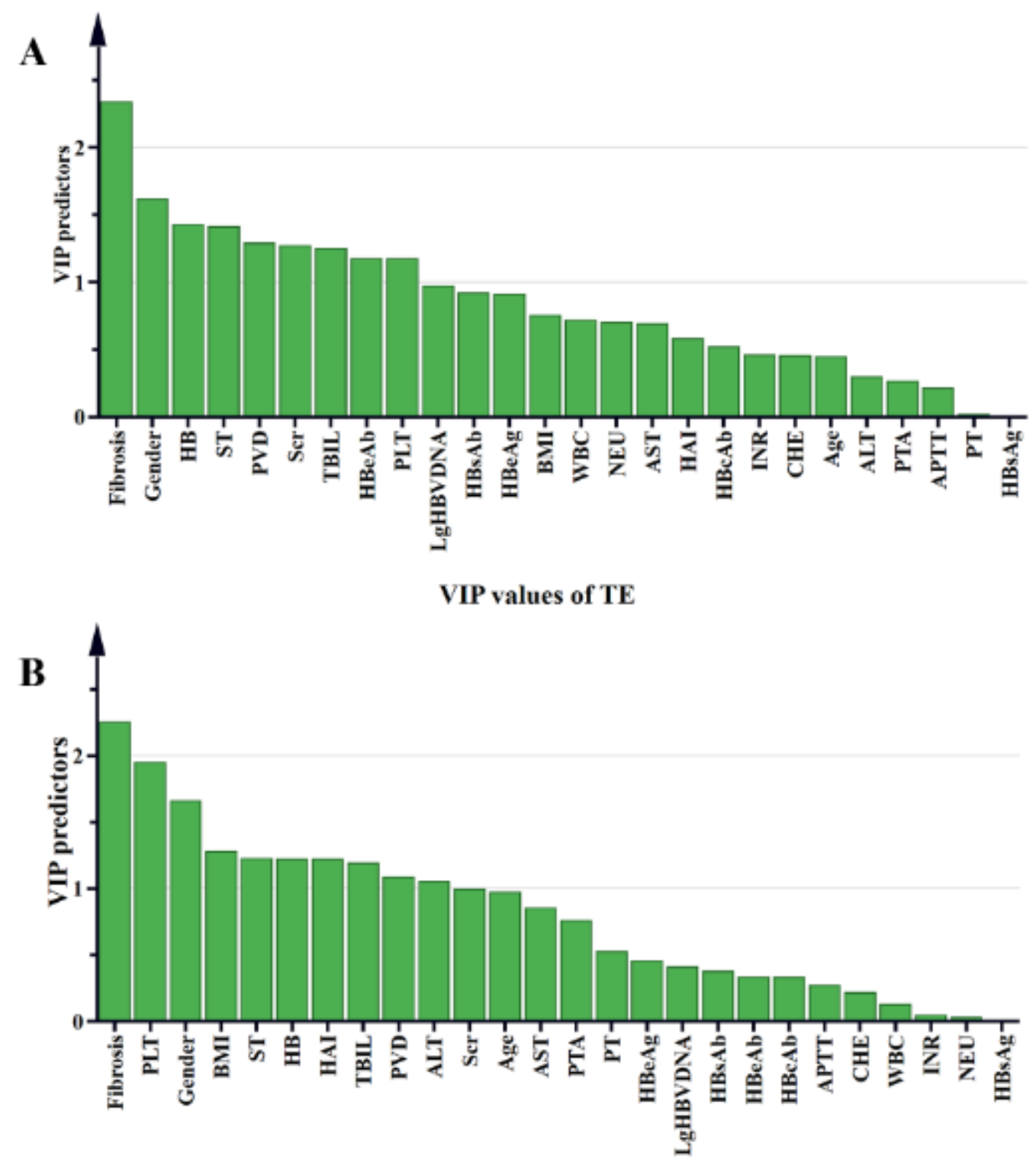

Figure 1

The results of orthogonal partial least squares (OPLS) discriminant analysis for SWE (A) and TE (B). The abscissa means various factors, it is arranged from left to right according to the influence to LSMs. The ordinate means the VIP value, the higher of the value represent the power affection. 


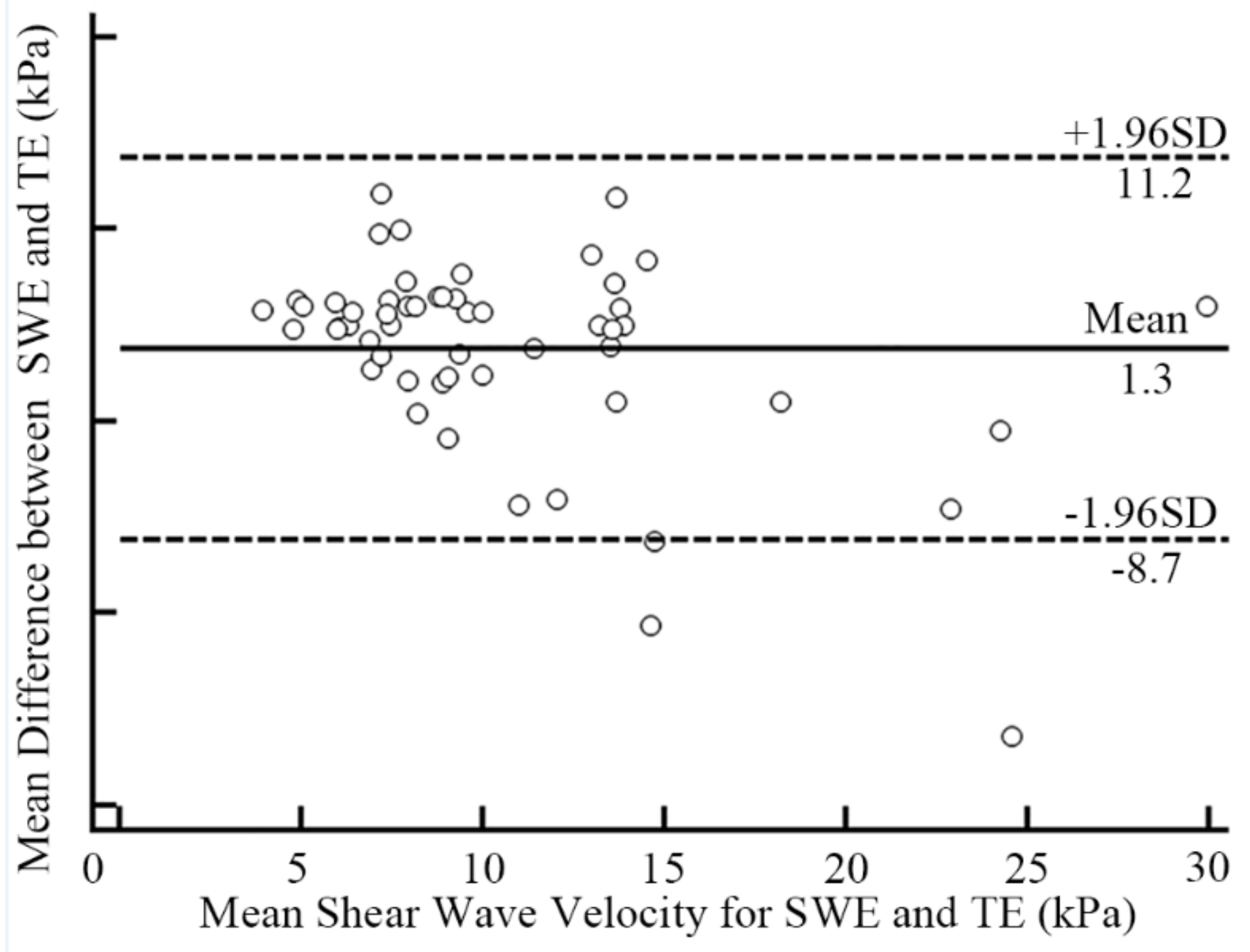

Figure 2

Bland-Altman analysis: Agreement between liver stiffness measurements (LSMs) obtained with shear wave elastography (SWE) and transient elastography (TE). The abscissa is the mean of the two measuring methods, the ordinate is the difference between the two measuring methods. The solid line represents the mean of the difference in shear wave velocity of SWE (A) and TE (B); the dash lines represent the $95 \%$ upper and lower limits of agreement, respectively. 
SWE

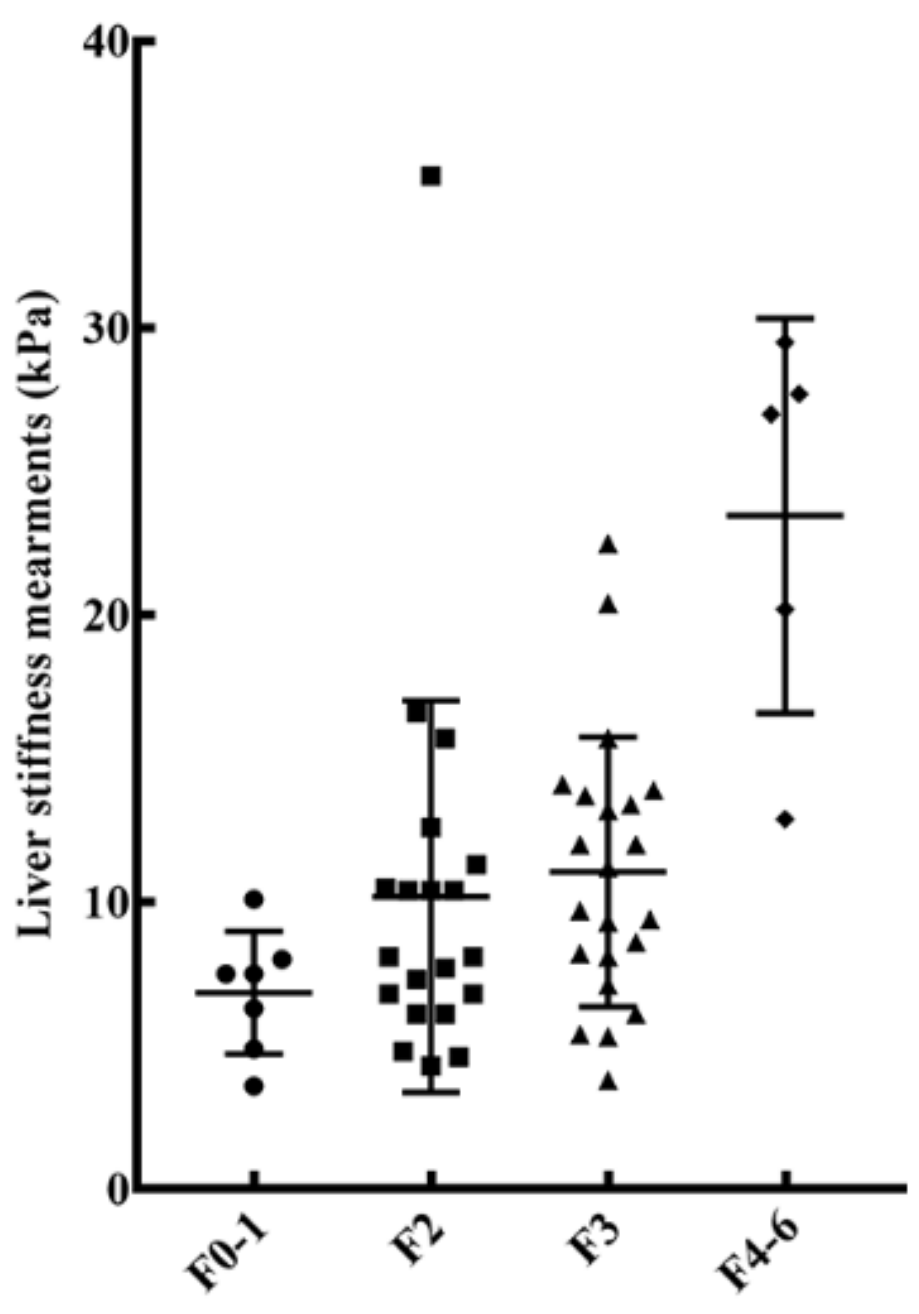

Liver fibrosis stages

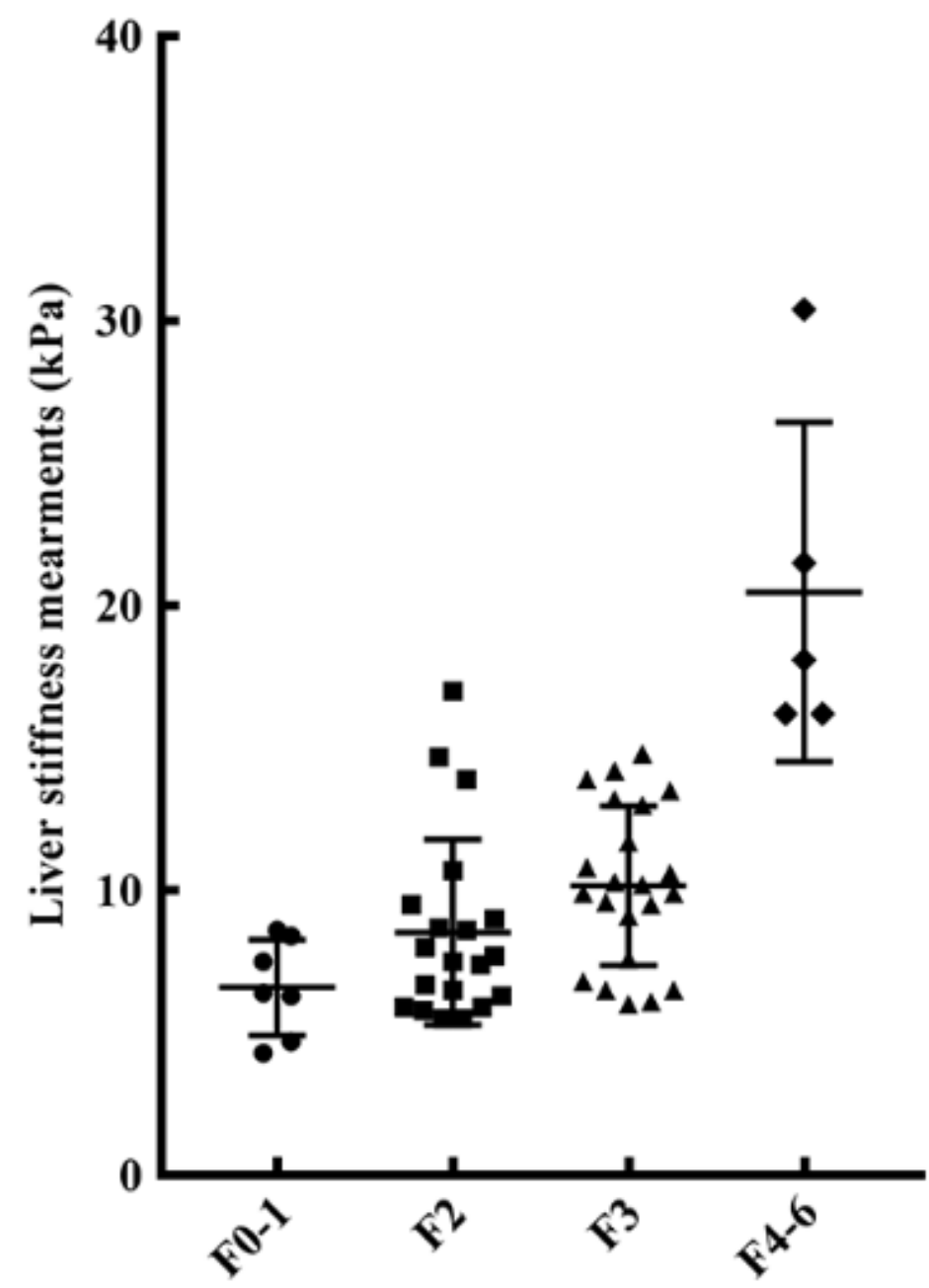

Liver fibrosis stages

Figure 3

Liver stiffness measurements (LSMs) obtained using shear wave elastography (SWE) and transient elastography (TE) in CHB patients. The long line indicates medians, the two short lines outside indicate the interquartile ranges. 


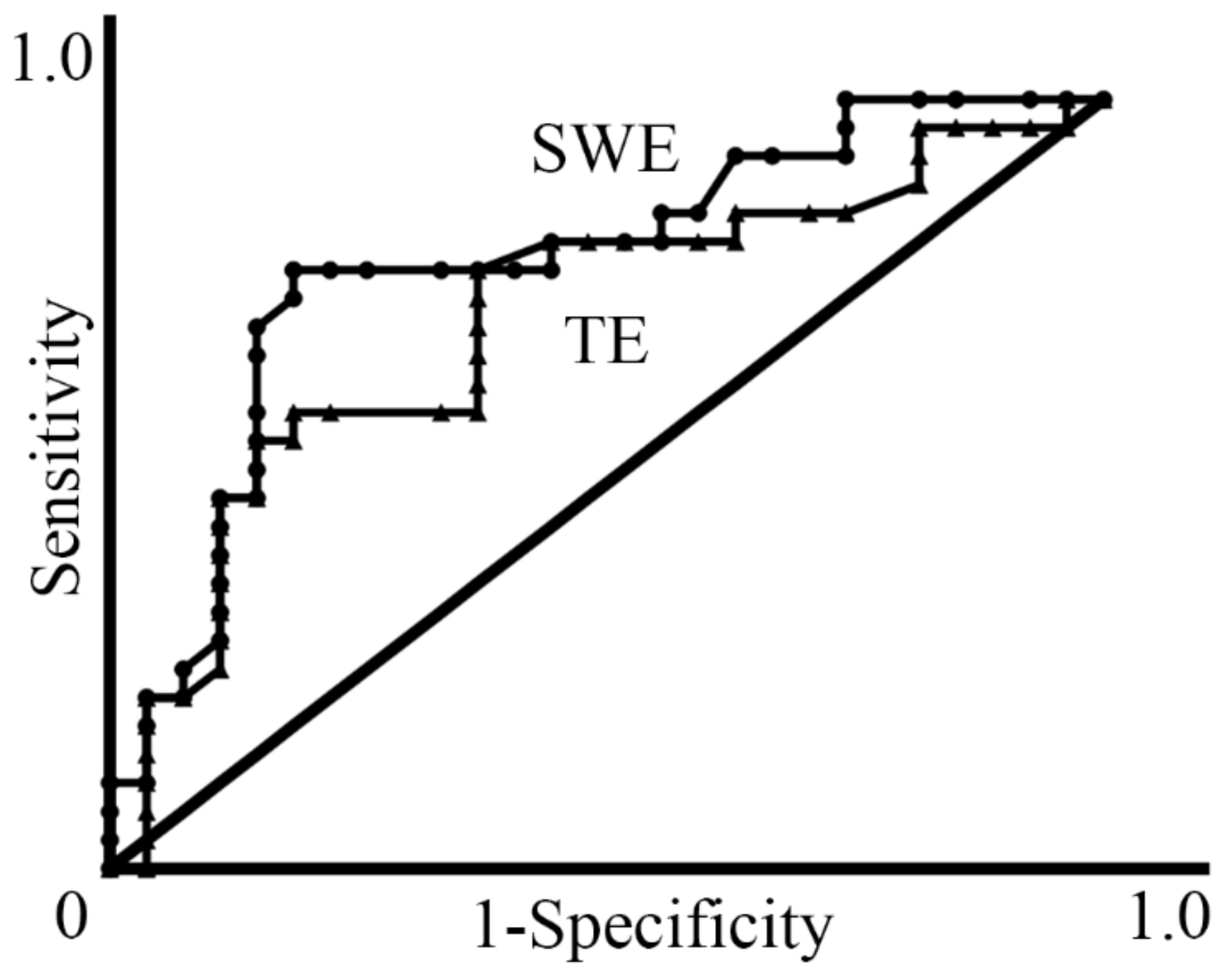

Figure 4

Area under the receiver operating characteristic curves (AUROCs) for shear wave elastography (SWE) and transient elastography $(T E)$ in assessing significant liver fibrosis $(F>2)$ in patients with chronic hepatitis $B$ infection. There were no significant differences in the AUROCs between the two examinations.

\section{Supplementary Files}

This is a list of supplementary files associated with this preprint. Click to download.

- Additionalfile1.docx 